\title{
Homology and symmetry breaking in Rayleigh-Bénard convection: Experiments and simulations
}

\author{
Kapilanjan Krishan, ${ }^{a)}$ Huseyin Kurtuldu, and Michael F. Schatz ${ }^{\text {) }}$ \\ Center for Nonlinear Science and School of Physics, Georgia Institute of Technology, \\ Atlanta, Georgia 30332, USA \\ Marcio Gameiro ${ }^{\mathrm{c})}$ and Konstantin Mischaikow ${ }^{\mathrm{c})}$ \\ School of Mathematics, Georgia Institute of Technology, Atlanta, Georgia 30332, USA \\ Santiago Madruga ${ }^{\text {d) }}$ \\ Engineering Science and Applied Mathematics, Northwestern University, Evanston, Illinois 60208, USA
}

(Received 8 June 2007; accepted 12 September 2007; published online 12 November 2007)

\begin{abstract}
Algebraic topology (homology) is used to analyze the state of spiral defect chaos in both laboratory experiments and numerical simulations of Rayleigh-Bénard convection. The analysis reveals topological asymmetries that arise when non-Boussinesq effects are present. The asymmetries are found in different flow fields in the simulations and are robust to substantial alterations to flow visualization conditions in the experiment. However, the asymmetries are not observable using conventional statistical measures. These results suggest homology may provide a new and general approach for connecting spatiotemporal observations of chaotic or turbulent patterns to theoretical models. (C) 2007 American Institute of Physics. [DOI: 10.1063/1.2800365]
\end{abstract}

\section{INTRODUCTION}

Recent technical advances in experimental fluid mechanics now make it possible to measure complex dynamical behavior with high resolution in space and time. ${ }^{1}$ Similarly, modern computational fluid dynamics methods permit modelling of complex chaotic and turbulent flows. ${ }^{2}$ The data sets produced by such experiments and simulations can be enormous; as a result, interpreting the results becomes a significant challenge. In particular, characterizing the geometric properties of complex spatiotemporal patterns in large data sets has been difficult because, to date, no general methodology has existed for extracting geometric signatures.

Algebraic topology provides a tool for describing global geometric properties of structures. Devised by Poincaré ${ }^{3}$ for use in global nonlinear analysis, algebraic topology originally used as input analytically defined objects (e.g., level sets of differentiable functions) to produce an output in the form of algebraic quantities which convey topological information about the input. In modern times, input objects can be expressed either as simplicial or cubical complexes. In fluid mechanics and in most fields of science and engineering, cubical representations often arise naturally in both experiments (raw image data represented as square pixels or cubic voxels) and simulations (fields computed at gridpoints on square or cubic lattices). A package of computer programs has been developed to perform computations of algebraic topology (computational homology) on cubical complexes in arbitrary dimensions. This suite of tools, called CHOMP

\footnotetext{
${ }^{a)}$ Present address: Department of Physics and Astronomy, University of California, Irvine, Irvine, CA 92697, USA.

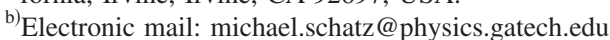

${ }^{c)}$ Present address: Rutgers University, New Jersey, USA.

${ }^{d)}$ Present address: Max-Planck-Institute for the Physics of Complex Systems, 01187 Dresden, Germany.
}

(Computational Homology Project) is freely available for download via the Web. ${ }^{4}$

We report here the first use of computational homology to characterize data obtained from a laboratory experiment. We analyze Rayleigh-Bénard convection in the state known as spiral defect chaos, ${ }^{5}$ which is widely considered a paradigm for the little understood phenomenon known as spatiotemporal chaos (see, for example, Ref. 6). In planform, patterns of spiral defect chaos, which are observed just above convective onset in low Prandtl number $(\sim 1)$ fluids, are composed of convection rolls deformed into numerous rotating spirals and riddled with dislocations, disclinations, and grain boundaries. Spiral defect chaos has been quantitatively described by a wide variety of approaches, including structure factors, correlation lengths and times as well as wave number, spectral, and spiral number distributions ${ }^{7}$ (see also Ref. 1 and references therein).

Thermal convection is frequently modeled using the Boussinesq approximation, which assumes that the temperature dependence of the fluid properties can be neglected, except for the temperature-induced density difference in the buoyant force that drives the flow. However, non-Boussinesq effects can arise in flows both in the laboratory and in natural settings. At convective onset, the subcritical bifurcation to hexagonal patterns is a clear signature of non-Boussinesq effects. ${ }^{8}$ (Straight convection rolls arise at onset from a supercritical bifurcation in Boussinesq Rayleigh-Bénard convection. ${ }^{9}$ ) Non-Boussinesq effects can be described quantitatively using perturbation theory near onset; in this regime they are characterized by parameter $Q$ introduced by Busse. ${ }^{8}$ Values of $Q \geqslant \sim 1$ indicate significant non-Boussinesq effects; $Q=0$ for Boussinesq convection. As changes in control parameter move the convective flow well away from onset, non-Boussinesq effects typically become more important and 

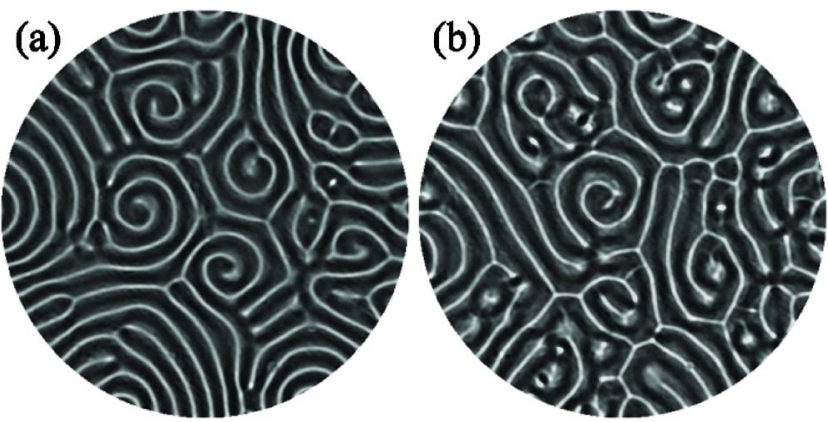

(c)

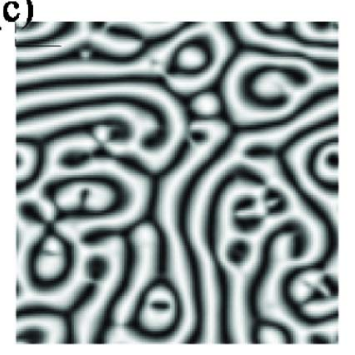

(d)

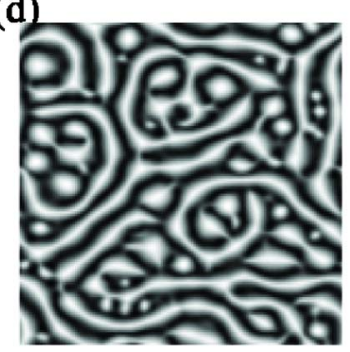

FIG. 1. (Color online) Images of spiral defect chaos convection are shown from laboratory experiments (a, b) and numerical simulations (c, d). Shadowgraph images from the experiments illustrate the convective flows at (a) $\epsilon=1.0$ and (b) $\epsilon=2.5$. The midplane temperature field is shown at $\epsilon=1.4$ for simulations carried out under (c) Boussinesq $(Q=0)$ and (d) non-Boussinesq $(Q=4.5)$ conditions. In all cases, dark regions in the images indicate the hot upflows and bright regions indicate cold downflows in the convective patterns.

are more difficult to characterize theoretically and experimentally.

\section{EXPERIMENTAL PROCEDURE}

We measure convective flow in a horizontal layer of compressed (3.2 $\mathrm{MPa}$ absolute pressure) $\mathrm{CO}_{2}$ gas of depth $d=0.0690 \pm 0.0005 \mathrm{~cm}$. The layer is bounded above by a $5 \mathrm{~cm}$ thick sapphire window and below by $1 \mathrm{~cm}$ thick goldplated aluminum mirror. The lateral walls are circular, formed out of an annular stack of filter paper sheets $3.80 \mathrm{~cm}$ in diameter. An electrical resistive heater is used to heat the bottom mirror to a temperature of $T_{h}$, and the top window is cooled to a fixed temperature of $T_{c}=21.20 \pm 0.02{ }^{\circ} \mathrm{C}$ by circulating chilled water. When the temperature difference, i.e., $\Delta T=T_{h}-T_{c}$, across the gas exceeds a critical temperature difference $\Delta T_{c}=4.0 \pm 0.1^{\circ} \mathrm{C}$, the onset of fluid motion occurs. The Prandtl number Pr is 0.97. In the experiments, the system control parameter, the reduced Rayleigh number $\epsilon$ $=\left(\Delta T-\Delta T_{c}\right) / \Delta T_{c}$, is increased above onset through a range where spiral defect chaos occurs. The characteristic time scale, the vertical diffusion time $t_{v}$, is approximately $2 \mathrm{~s}$.

The flows are visualized using the shadowgraph technique. ${ }^{10}$ Time series of shadowgraph images [Figs. 1(a) and $1(\mathrm{~b})]$ with a spatial resolution of $515 \times 650$ pixels are captured under computer control at a rate of $11 \mathrm{~Hz}$ using a 12-bit digital camera interfaced to a frame grabber. The images are prepared for analysis by subtracting a background image of the fluid below onset and using digital Fourier filtering to remove high wave number components due to cam-

era spatial noise. The median value of intensity for all pixels in the image is then determined and used as a typical threshold to characterize each pixel as describing either hot upflow or cold downflow in the convection pattern. The resulting time series of thresholded 1-bit images are used for computing homology.

\section{NUMERICAL SIMULATIONS}

Our direct numerical simulations of the Boussinesq equations employ a pseudospectral code developed by Pesch and co-workers. ${ }^{11,12}$ The code uses Fourier modes in the horizontal direction and appropriate combinations of trigonometric and Chandrasekhar functions that satisfy the top and bottom boundary conditions in the vertical direction. ${ }^{8}$ All runs are performed with six vertical modes and $128 \times 128$ horizontal Fourier modes in a square domain with side length equal to 16 times the pattern wavelength at convective onset. The time step is typically $\tau_{v} / 500$. For our analysis, the flows are represented by $128 \times 128$ images [Figs. 1(c) and $1(\mathrm{~d})$ ] of the temperature field or the vertical velocity component. The images are typically stored every $2 \tau_{v}$. The median value of the flow field quantity (temperature or vertical velocity) for each image is determined and used as a threshold to characterize each gridpoint as describing hot upflow or cold downflow. Thus, as in the laboratory experiment, the resulting time series of thresholded 1-bit images are used for computing homology.

In the simulations, we describe non-Boussinesq effects arising from the temperature dependence of material properties by a Taylor expansion truncated at leading order beyond the Boussinesq approximation. The simulations are performed at constant mean temperature $\left(T_{h}+T_{c}\right) / 2$; the expansion is carried out about the mean temperature. In this case, the parameter $Q$ (see Ref. 8) is given by

$$
Q=\sum_{i=0}^{4} \gamma_{i}^{c} \mathcal{P}_{i},
$$

where the quantities $\mathcal{P}_{i}$ are linear functions of $\operatorname{Pr}^{-1}$, and the non-Boussinesq coefficients $\gamma_{i}^{c}$ give the difference of the respective fluid properties across the layer at threshold $(\epsilon=0)$. For simulations away from onset $(\epsilon>0)$, the $\epsilon$ dependence of non-Boussinesq effects is characterized by coefficients $\gamma_{i}=\gamma_{i}^{c}(1+\epsilon)$. (See Ref. 13 for more details.) In nonBoussinesq simulations, all the $\gamma_{i}^{c}$ are retained, while in the Boussinesq simulations, $\gamma_{i}^{c}$ are set to 0 . In all simulations, we fix $\epsilon=1.4$ and set $\operatorname{Pr}=0.8$.

\section{RESULTS}

Formally, homology is computed for a topological space $X$ of $N$ dimensions by systematically assigning a sequence of Abelian groups $H_{k}(X)(k=0,1,2, \ldots, N-1)$ to $X$. For our purposes, it is sufficient to take $H_{k}$ to be products of the integers, i.e., $H_{k}(X)=Z^{\beta_{k}(X)}$, where the integer dimensions of the groups $\beta_{k}(X) \geqslant 0$ are also known as the Betti numbers. In this work we focus solely on $\beta_{k}(X)$ as the output of the homological analysis; each $\beta_{k}(X)$ describes a topological 


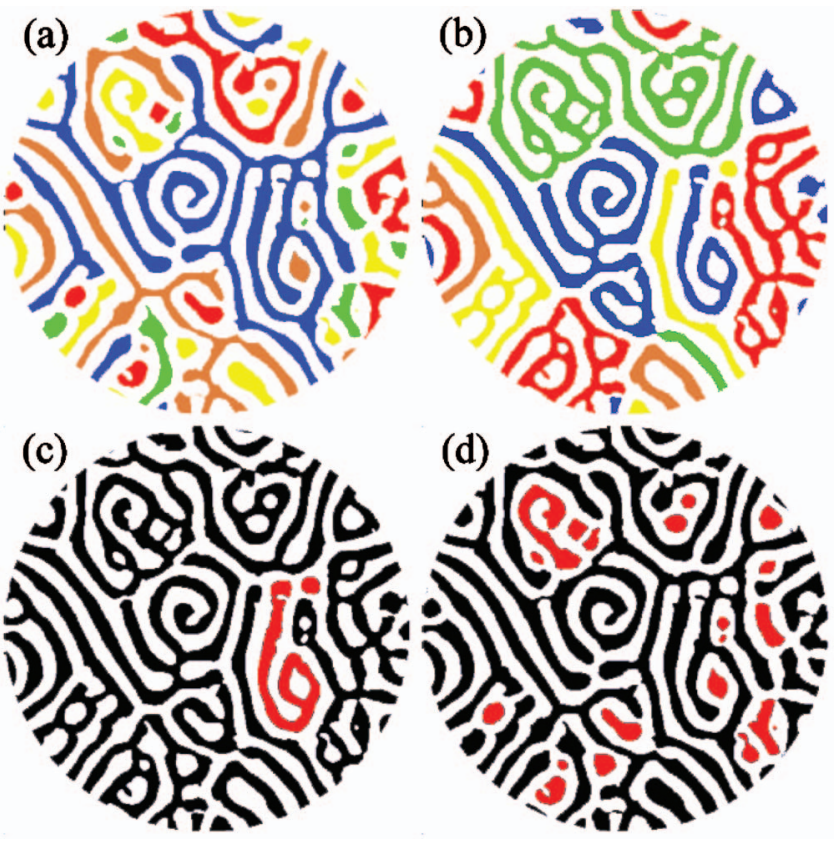

FIG. 2. (Color) Computation of the homology for the experimental data in Fig. 1(b) yields a sequence of Betti numbers $\beta$, which can be readily interpreted visually. The number of distinct components is indicated by the zeroth Betti number for cold downflows (a) $\beta_{0 c}=49$ and for hot upflows (b) $\beta_{0 h}=24$. (Different colors are used in (a) and (b) to distinguish a given component from its nearest neighbors.) The number of holes is given by the first Betti number for cold downflows (c) $\beta_{1 c}=2$ and for hot upflows (d) $\beta_{1 h}=20$. (Each hole is colored red in (c) and (d).)

property of $X$. Thus, the net result of this analysis is the characterization of $X$ by a set of $N$ non-negative integers. (See Ref. 4 for more details.)

From each 1-bit image in time series from either experiments or simulations, two distinct, topological spaces are obtained: $X_{h}$, where the hot upflow pixels have a nonzero value, and $X_{c}$, where the cold downflow pixels have a nonzero value. $X_{c}$ and $X_{h}$, which are two-dimensional, are input, in turn, into the homology codes, which subsequently output two Betti numbers for each space: $\beta_{0 h}, \beta_{1 h}$ for the hot upflows, and $\beta_{0 c}, \beta_{1 c}$ for the cold downflows. $\beta_{0 h}\left(\beta_{0 c}\right)$ counts the number of distinct components; i.e., the number of regions of hot upflow (cold downflow) that are separated from similar regions in a given pattern $X_{h}\left(X_{c}\right)$ [Figs. 2(a) and 2(b)]. $\beta_{1 h}\left(\beta_{1 c}\right)$ counts the number of holes in the hot upflows (cold downflows) in a given pattern $X_{h}\left(X_{c}\right)$ [Fig. 2(c) and 2(d)]. With the package CHOMP, computing the homology of $X_{c}$ and $X_{h}$ corresponding to each image takes about $1 \mathrm{~s}$ on a $2.2 \mathrm{Ghz}$ CPU.
Figure 2 shows a striking result: in the experiments, hot upflows are topologically quite distinct from cold downflows. Specifically, there are more cold downflow components than hot upflow components $\left(\beta_{0 c}>\beta_{0 h}\right)$. Moreover, the hot upflow regions contain more holes than the cold downflow regions $\left(\beta_{1 h}>\beta_{1 c}\right)$. This distinction is not revealed using standard statistical measures of the pattern. For example, the mean area occupied by upflow is equal to that occupied by downflow by construction (when the threshold is set to the median pixel intensity in the original image.) Wave number distributions obtained from Fourier analysis of $X_{h}$ and $X_{c}$ show no discernible differences.

These measurements of topology are robust to variations in the choice of threshold. The choice of the median pixel intensity as the threshold to separate upflows from downflows is physically well-motivated but somewhat arbitrary. In practice, any reasonable choice yields similar results. For example, for $X_{h}$ and $X_{c}$ in Fig. 2, choosing the mean pixel intensity (which is larger than the median intensity by approximately $5 \%$ of full scale) as the threshold yields nearly identical Betti numbers: $\beta_{0 h}=22, \beta_{0 c}=53, \beta_{1 h}=23$, and $\beta_{1 c}$ $=3$.

Time series of the Betti numbers exhibit fluctuations about well-defined time-average values (Fig. 3). The fluctuations are primarily a global signature of the complex spatiotemporal behavior of spiral defect chaos. Mean flow induced by curvature in the roll pattern leads to regions of local compression or dilatation throughout the pattern. Compression often leads to merging of neighboring rolls, while the dilatation results in the formation of a new rolls in the pattern; these processes are closely related to secondary instability mechanisms for ideal straight rolls. ${ }^{1,8}$ These local events drive further changes in pattern curvature, thereby leading to a continually evolving pattern with fluctuating topology. The Betti numbers are a global measure of the topological changes, and therefore, are dependent on the local processes, for which theories of defect dynamics have been proposed. ${ }^{14}$ How Betti numbers are related to defect dynamics remains an open question; for our purposes, we focus on the time-average values of the Betti numbers $\left(\bar{\beta}_{0 h}, \bar{\beta}_{1 h}, \bar{\beta}_{0 c}, \bar{\beta}_{1 c}\right)$, which we find to be stationary for fixed $\epsilon$.

The measurements of $\bar{\beta}$ are robust with respect to flow visualization conditions. It is well known that shadowgraphy can introduce significant nonlinearities and image artifacts (e.g., caustics); the strength of these effects depend on the effective optical distance $z_{1}$ of the shadowgraph system. ${ }^{10}$ (a)

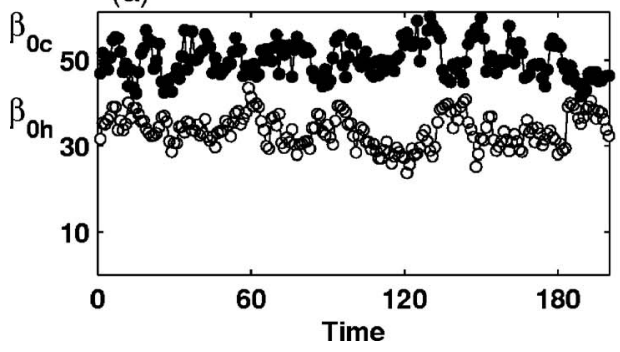

(b)

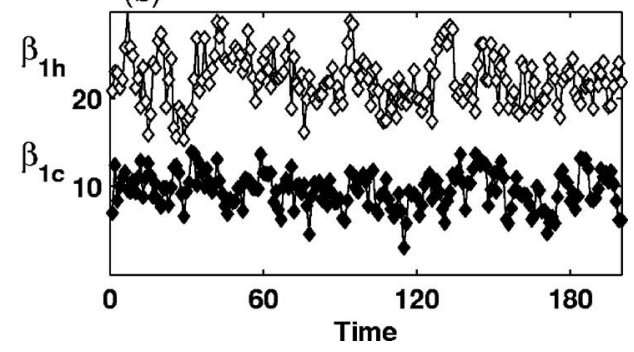

FIG. 3. Time series of (a) the zeroth Betti numbers $\beta_{0 h}$ (open circles) and $\beta_{0 c}$ (filled circles), and (b) the first Betti numbers $\beta_{1 h}$ (open diamonds) and $\beta_{1 c}$ (filled diamonds) are obtained from laboratory experiments at $\epsilon=2.5$. Time is scaled by $t_{v}$; the time interval between samples is $t_{v} / 2$. 


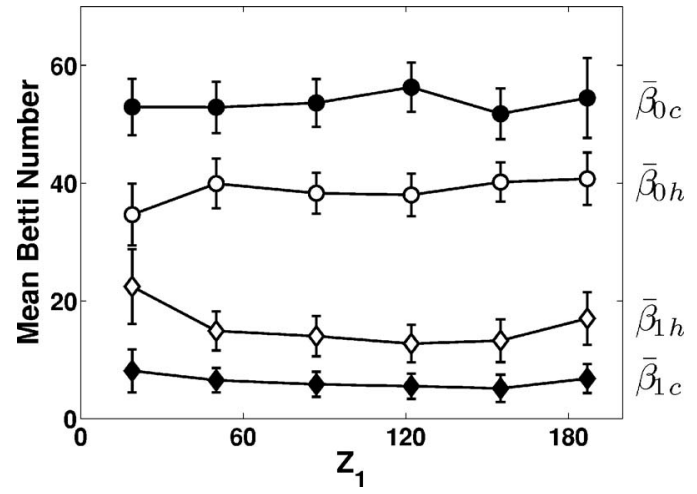

FIG. 4. The mean Betti numbers are plotted as a function of the effective optical distance $z_{1}$ of the shadowgraph system in laboratory experiments performed at $\epsilon \cong 2$. For each data point, the median pixel intensity of the raw shadowgraph images was used as the threshold for the homology analysis.

We have checked for possible sensitivity to shadowgraphy visualization by conducting a series of experiments where the conditions of the convective flow were fixed and image time series were captured for different values of $z_{1}$. Figure 4 shows that the mean Betti number changes only slightly as $z_{1}$ is varied over nearly an order of magnitude. Additional experimental data (not shown) demonstrate that a change of sign in $z_{1}$ [which changes hot upflows (cold downflows) from bright (dark) to dark (bright)] does not affect the determination of $\bar{\beta}$.

The differences between the mean Betti numbers for hot upflows $\bar{\beta}_{0 h}, \bar{\beta}_{1 h}$ and for cold downflows $\bar{\beta}_{0 c}, \bar{\beta}_{1 c}$ become more substantial as $\epsilon$ increased above convective onset (Fig. 5). For $\epsilon<2.0$, the mean numbers of components and holes

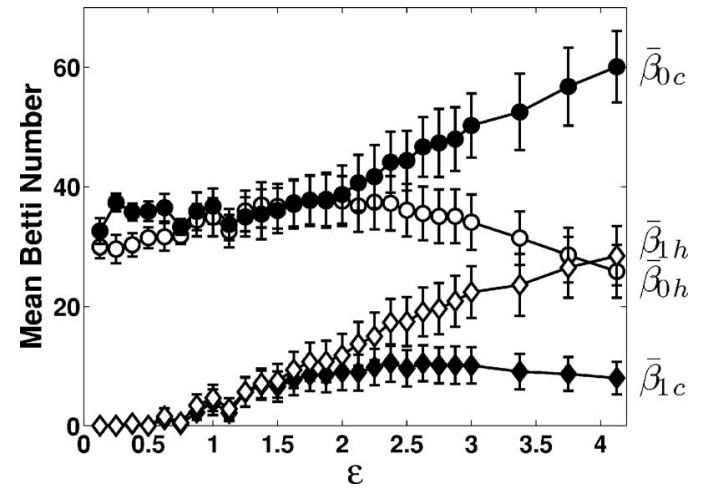

FIG. 5. The mean zeroth Betti numbers $\bar{\beta}_{0 h}$ (open circles) and $\bar{\beta}_{0 c}$ (filled circles), and first Betti numbers $\bar{\beta}_{1 h}$ (open diamonds) and $\bar{\beta}_{1 c}$ (filled diamonds) are shown as a function of $\epsilon$ for data from laboratory experiments. Each data point is obtained by averaging the Betti numbers from analysis of 18000 images corresponding to an observation time of approximately $1800 t_{v}$.

are roughly equal for both upflows and downflows. Moreover, the number of holes in downflows/upflows is effectively zero for $\epsilon<0.7$, where the patterns consist essentially of straight rolls. Near the onset of spiral defect chaos (at approximately $\epsilon=0.7$ in our experiment, the number of holes for upflows/downflows becomes nonzero. For $\epsilon>2.0$, the difference in the average component number grows significantly as both the number of cold components grows and the number of hot components shrinks. The behavior in the number of holes is somewhat different; for $\epsilon<2.0$, the number of holes increases significantly in the hot upflows but decreases only weakly for the cold downflows. (a)
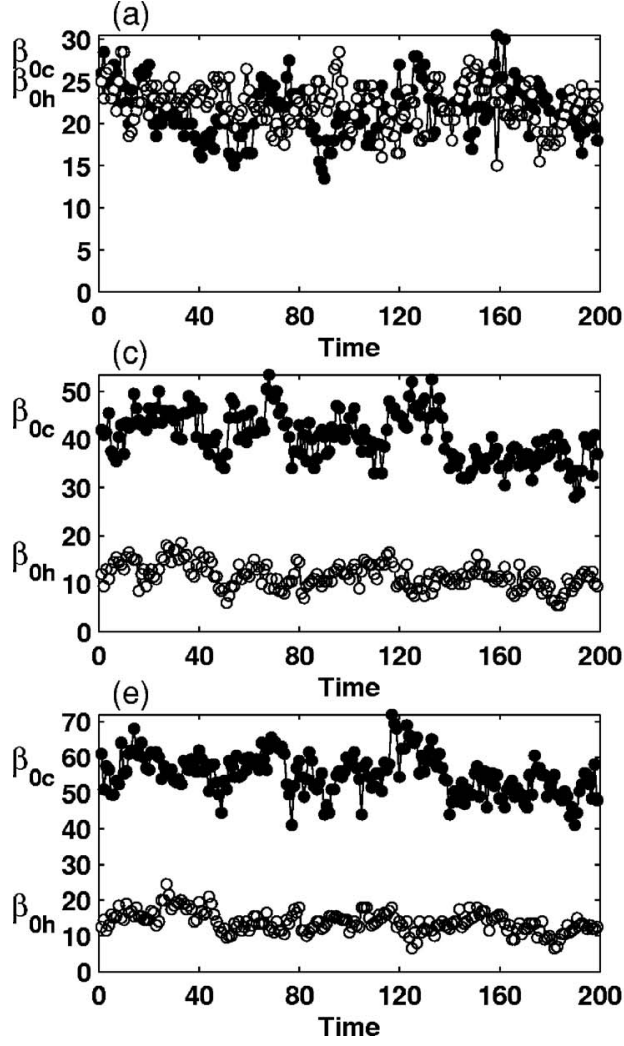

(b)
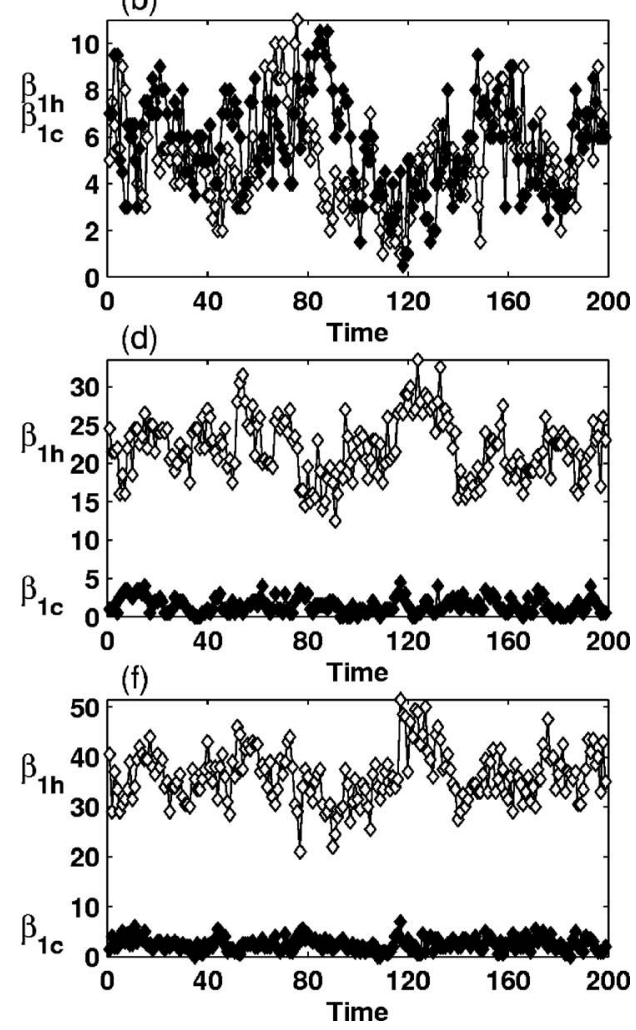

FIG. 6. Time series of the zeroth Betti numbers $\beta_{0 h}$ (open circles) and $\beta_{0 c}$ (filled circles), and the first Betti numbers $\beta_{1 h}$ (open diamonds) and $\beta_{1 c}$ (filled diamonds) are obtained from numerical simulations at $\epsilon=1.4$. The midplane temperature field from Boussinesq simulations is used to obtain time series of (a) $\beta_{0 h}, \beta_{0 c}$ and (b) $\beta_{1 h}, \beta_{1 c}$. The midplane temperature field from non-Boussinesq simulations is used to obtain time series of (c) $\beta_{0 h}$, $\beta_{0 c}$ and (d) $\beta_{1 h}, \beta_{1 c}$. The vertical velocity component at $z=-0.25$ from non-Boussinesq simulations is used to obtain time series of (e) $\beta_{0 h}, \beta_{0 c}$ and (f) $\beta_{1 h}, \beta_{1 c}$. (The midplane is located at $z=0$ and the bottom boundary is located at $z=-0.5$.) Time is scaled by $t_{v}$. 
These experimental observations, taken as a whole, suggest the observed asymmetries in the Betti numbers may be due to the breakdown of the Boussinesq approximation (and corresponding breaking of the Boussinesq symmetry). To check this hypothesis, we conducted two simulations under identical conditions except one simulation was Boussinesq $(Q=0)$ and the other simulation was non-Boussinesq $(Q$ =4.5). The Betti number time series for the Boussinesq simulation shows little distinction in the Betti numbers [Figs. 6(a) and 6(b)]. By contrast, examination of the same field variable in non-Boussinesq simulation [Figs. 6(c) and 6(d)] shows distinct differences in the Betti numbers that are qualitatively in agreement with the experimental observations.

Different flow fields extracted from the simulation exhibit similar qualitative behavior in the time-averaged Betti numbers [Figs. 6(c)-6(f)]. We examined both the temperature field and the vertical velocity components sampled at the vertical positions $z=0$ (the midplane of the convection cell), $z=-0.25$ and $z=+0.25$. (The top and bottom boundaries are located at $z=+0.5$ and $z=-0.5$, respectively.) The quantitative values for the time-averaged Betti numbers differ weakly between different projections of the convective flow. However, every projection exhibits the same qualitative result; namely, in a given projection of Boussinesq convection, the time-averaged Betti numbers for hot upflows and cold downflows are the same, while each projection of nonBoussinesq exhibits the same Betti number asymmetries.

\section{CONCLUSIONS}

We conclude that the breakdown of the Boussinesq approximation can be readily observed in data from convection experiments and simulations by analyzing the topology using computational homology. It might be argued the use of homology constitutes an "excessive use of force" for the twodimensional patterns analyzed here since the counting of features such as components and holes could be accomplished by other means. Nevertheless, these upflow/downflow topological asymmetries, which had remained unnoticed despite decades of experimental study of convective flows, were uncovered by a systematic analysis suggested by the homology formalism. Moreover, the homology analysis outlined here can be readily extended to higher dimensions where less sophisticated approaches will likely fail. For example, threedimensional complexes can be formed from the image data used here by creating "time-blocks" of data with two spatial dimensions and one time dimension; such data are expected to contain new topological features that capture dynamical information. $^{15}$

Our results are consistent with well-known symmetries/ asymmetries of convective flows that arise at onset. Physically, non-Boussinesq effects at onset are commonly associated with the temperature dependence of the fluid's physical properties. ${ }^{8}$ In particular, for convection in gases considered here, the kinematic viscosity typically increases with increasing temperature; this particular temperature dependence leads to stable flow at onset in the form of "down-hexagons," which have cold downflow in the center of each hexagon. The zeroth Betti numbers of a pattern containing $M$ hexago- nal convection cells in an ordered array can be easily determined. In gas convection, the cold downflow at the center of each hexagon will be isolated for all other cold downflows, yielding $\beta_{0 c}=M$, while the hot upflows around the edges of all hexagons will be connected, yielding $\beta_{0 h}=1$. This Betti number asymmetry $\left(\beta_{0 c}>\beta_{0 h}\right)$ is consistent with the results for our non-Boussinesq experiments and simulations far from onset, where the temperature-dependent variation of fluid properties is of larger magnitude but has the same "sign" as the variations near onset. Based on these considerations, we conjecture that a homological analysis of farfrom-onset non-Boussinesq flows in typical liquids (e.g., water) should exhibit the Betti number asymmetry $\beta_{0 h}>\beta_{0 c}$, since this asymmetry would be consistent with the typical appearance of "up-hexagons" at onset of non-Boussinesq convection in such fluids. (The physical origin up-hexagons in liquids can be traced to the typical temperature dependence of the kinematic viscosity, which usually decreases with increasing temperature.)

Our results suggest that computational homology might be a useful tool in a wide variety of cases in fluid dynamics. For example, in the atmospheric sciences, where extensive use is made of the Boussinesq approximation, homological analysis may provide new ways to characterize atmospheric data. The use of homology need not be limited to convection; this approach may be applied in any fluid flow where quantitative characterization of complex data is needed.

\section{ACKNOWLEDGMENTS}

We thank G. Ahlers, G. Gunaratne, B. Kalies, and H. Riecke for their helpful comments about this work; we also thank W. Pesch for generously providing the code used for numerical work presented in this paper. This work was supported by DARPA (K.M. and M.G.), the Department of Energy under Grant No. 97891, and the National Science Foundation under Grant Nos. DMS-0107396 (K.M. and M.G.), CTS-0201610 (M.F.S. and K.K.), DMS-0443827 (K.M.), ATM-0434193 (M.F.S. and H.K.), and DMS-0511115 (K.M.). In addition, M.G. acknowledges the support of a CAPES (Brazil) fellowship, and S.M. acknowledges EU support under Grant No. MRTN-CT-2004-005728.

${ }^{1}$ E. Bodenschatz, W. Pesch, and G. Ahlers, "Recent developments in Rayleigh-Bénard convection," Annu. Rev. Fluid Mech. 32, 709 (2000).

${ }^{2}$ G. Kawahara and S. Kida, "Periodic motion embedded in plane Couette turbulence: regeneration cycle and burst," J. Fluid Mech. 449, 291 (2001).

${ }^{3}$ H. Poincaré, “Analysis situs,” J. Ec. Polytech. (Paris) 1, 1 (1895).

${ }^{4}$ T. Kaczynski, K. Mischaikow, and M. Mrozek, Computational homology, Applied Mathematical Sciences (Springer-Verlag, New York, 2004), Vol. 157.

${ }^{5}$ S. W. Morris, E. Bodenschatz, D. S. Cannell, and G. Ahlers, "Spiral defect chaos in large aspect ratio Rayleigh-Bénard convection," Phys. Rev. Lett. 71, 2026 (1993).

${ }^{6}$ J. P. Gollub, "Pattern-formation: Spirals and chaos," Nature 367, 318 (1994).

${ }^{7}$ H. Riecke and S. Madruga, "Geometric diagnostics of complex patterns: Spiral defect chaos," Chaos 16, 013125 (2006).

${ }^{8}$ F. H. Busse, "The stability of finite amplitude cellular convection and its relation to an extremum principle," J. Fluid Mech. 30, 625 (1967).

${ }^{9}$ A. Schlüter, D. Lortz, and F. H. Busse, "On the stability of steady finite amplitude convection,” J. Fluid Mech. 23, 129 (1965). 
${ }^{10}$ J. R. de Bruyn, E. Bodenschatz, S. W. Morris, S. P. Trainoff, Y. Hu, D. S. Cannell, and G. Ahlers, "Apparatus for the study of Rayleigh-Bénard convection in gases under pressure," Rev. Sci. Instrum. 67, 2043 (1996).

${ }^{11}$ W. Pesch, "Complex spatiotemporal convection patterns," Chaos 6, 348 (1996).

${ }^{12}$ W. Decker, W. Pesch, and A. Weber, "Spiral defect chaos in RayleighBénard convection," Phys. Rev. Lett. 73, 648 (1994).
${ }^{13}$ S. Madruga, H. Riecke, and W. Pesch, "Re-entrant hexagons in nonBoussinesq convection," J. Fluid Mech. 548, 341 (2006).

${ }^{14}$ M. C. Cross, and P. C. Hohenberg, "Pattern formation outside of equilibrium," Rev. Mod. Phys. 65, 851 (1993).

${ }^{15}$ M. Gameiro, K. Mischaikow, and W. Kalies, "Topological characterization of spatial-temporal chaos," Phys. Rev. E 70, 035203 (2004). 